\title{
Error Analysis of 2-tier $M$-ary Star QAM Modulation in Shadowed Fading Channels
}

\author{
Sourjya Dutta \\ Polaris Networks \\ Salt Lake Electronics Complex \\ Kolkata 700091, India
}

\author{
Iti Saha Misra \\ Deptt. of Electronics and Telecommunication Engg. \\ Jadavpur University \\ Kolkata 700032, India
}

\begin{abstract}
The error performance of the 2-tier star shaped Quadrature Amplitude Modulation scheme over $K$ and $K_{G}$ fading channels are analyzed and evaluated. Novel closed form expressions for Symbol Error Rate (SER) have been derived for $M$-ary 2-tier circular Star QAM transmitted over the $K$ and $K_{G}$ fading channels. The expressions derived are in the form of sum of single definite integrals of hypergeometric functions which are calculated using numerical methods. The expressions are validated by extensive Monte Carlo simulation. A simple relationship between SER and bit error rate (BER) is proposed and experimentally verified. Using the expressions for SER, the optimum values of ring ratio are calculated for various values of $M$. It has also been established that the error performance of 2-tier Star QAM is considerably superior to that of the $M$-ary Square QAM for high and moderate fading.
\end{abstract}

\section{General Terms:}

Digital Modulation, Wireless Channel

\section{Keywords:}

Star Quadrature Amplitude Modulation (QAM), Rayleigh-Gamma $(K)$ Nakagami-Gamma $\left(K_{G}\right)$, Fading, Shadowing, Symbol Error Rate(SER), Bit Error Rate(BER)

\section{INTRODUCTION}

Various statistical models are used to study the effects of multipath fading and shadowing that severely degrade the performance of wireless communication systems. Effects of small-scale multipath fading on communication systems have been widely studied. The analysis of the effect of shadowing in wireless communication systems had been impeded by the complex mathematical form of the log-normal distribution conventionally used to quantify this phenomenon.

In relatively recent works the $K$ and the $K_{G}$ fading models have been found to be a suitable replacement of the log-normal distribution to estimate the combined effects of shadowing and small-scale fading in wireless media [1, 2, 3]. Moreover the Generalized- $K$ distribution is also being used to model the effect of turbulence in free space optical (FSO) communication channels [4 5]. The mathematical forms of these distributions allow closed form integration and hence system parameters can be efficiently computed using numerical techniques. Error analysis of lower order $M$-ary Phase Shift Keying (PSK) and square $M$-ary Quadrature Amplitude Modulation (MQAM) schemes for $K$ and $K_{G}$ channels is found in [3, 6, 7]. Similar analysis for higher order and complex modulation schemes are absent in literature.

$M$-ary QAM schemes are spectrally efficient modulation schemes which ensures higher data rates without requiring extra bandwidth. It has been pointed out in [8] that the widely used square $M$ QAM scheme will have a high chance of false phase locking in channels where both amplitude and phase of the transmitted signal may vary considerably. The authors in [8] have contended that the $M$-ary Star QAM modulation scheme will be better suited for vehicular environments. In [9] it has been shown that the $M$-ary Star QAM scheme gives a better performance in heavy fading channels like the Rayleigh channel and also simplifies the receiver structure as automatic gain control (AGC) and carrier recovery are no longer required. As given in [10], the $M$-ary Star QAM overcomes the problem of high peak-to-average power ratio (PAPR) present in square and rectangular QAM schemes. In recent academic literature [11, 12, 13, 14] the Star QAM modulation has been studied for applications in optical communication. Additionally in [15] it has been pointed out that circular QAM schemes provide better performance than rectangular QAM for quantum detection. Error analysis of Star MQAM schemes in small-scale multipath fading channels can be found in [16, 17, 18] but to the best of our knowledge error rate estimation of Star QAM in shadowed fading channels is absent in current literature. The aim of this paper is to analyse the combined effects of shadowing and multi-path fading on the performance of 2-tier $M$-ary Star QAM schemes. The contributions of this paper are :

i. Derivation of numerically computable expressions for average SER of Star MQAM considering the effects of both multipath fading and shadowing.

ii. Derivation of an efficient relationship between average SER and average BER for the 2-tier Star QAM.

iii. Proposing optimum values of ring ratio for different constellation sizes in composite multi-path and shadow fading channel.

iv. To compare the error performance of the Star and the Square constellations in the discussed propagation model.

The rest of the paper has been structured as follows. In Section 2 the channel models used are discussed. The theoretical derivation 


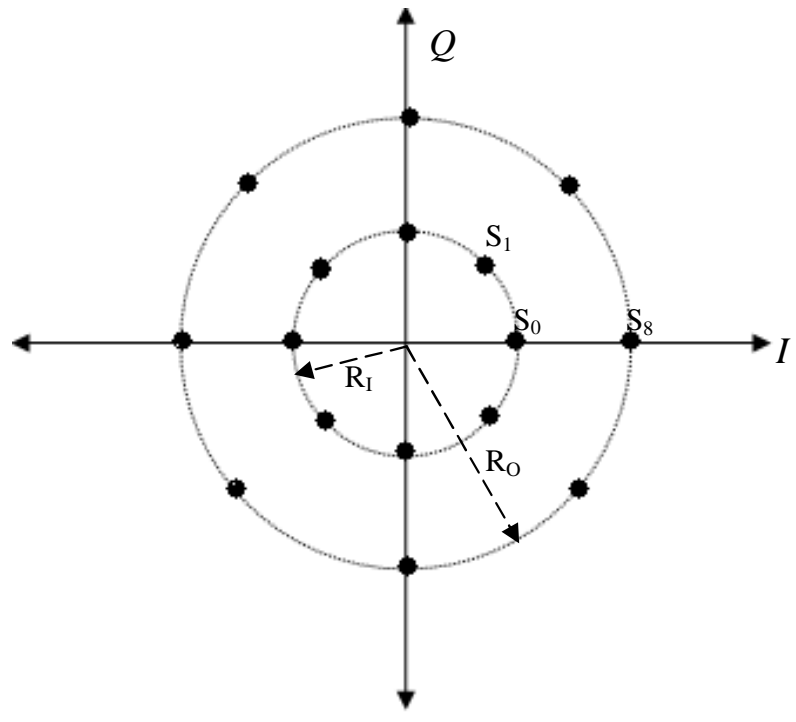

Fig. 1. Signal constellation of 2-tier 16-ary Star QAM.

of the average SER in $K$ and $K_{G}$ fading channel is shown in Section 3 The relationship between SER and bit error rate (BER) is given in Section 4 The optimum values of ring ratio for the Star QAM scheme are presented in Section 5 Numerical results and discussions are presented in Section 6, Section 7 concludes the paper.

\section{SYSTEM MODEL}

\subsection{2-tier Star MQAM Scheme}

The 2-tier $M$-ary Star QAM scheme, as shown in Figure 1 has the signalling points distributed over two amplitude levels $R_{I}$ and $R_{O}$ and on each amplitude level there are $M / 2$ signalling points which are placed at a constant phase difference $(4 \pi / M)$. Thus a signalling point $\left(s_{i}\right)$ of a Star QAM constellation can be given as,

$$
s_{i}=r_{i} \cdot e^{j \theta_{i}}
$$

where,

and

$$
r_{i} \in\left\{R_{I}, R_{O}\right\}
$$

$$
\theta_{i}=\frac{4 \pi i}{M}, i=0,1, \ldots, \frac{M}{2} .
$$

The signalling points are uncoded, i.e. for the 16-QAM in Figure 1 $s_{0}=0000, s_{1}=0001, s_{8}=1000$ and so long. The modulated wave is baseband transmitted over the wireless channel.

\subsection{Channel Model}

The uncoded dual ring $M$-ary Star QAM signal is transmitted over a wireless channel along with AWGN. The signal in this wireless channel is corrupted due to both short-term (multipath) and long-term (shadow) fading. The short-term fading is assumed to be slow compared to the signal variation. The received baseband signal can be given by $z=s X+n$ where $s$ is the transmitted symbol, $X$ is a random variable following a fading statistics $f_{\gamma}(\gamma)$ and $n$ is the Gaussian distributed additive noise.

When only channel degradation due to short term fading is considered, the probability density function of the signal amplitude can be given by $f_{\gamma}(\gamma)$ where $f_{\gamma}(\gamma)$ can be the Rayleigh distribution or the Nakagami- $m$ distribution given in [19]. When long term fading is considered, the power of the envelope which is assumed to be constant for multipath fading varies according to the lognormal distribution $f_{Y}(y)$. Thus the joint pdf of the random variable $X$ is given as,

$$
f_{X}(\gamma)=\int_{0}^{\infty} f_{\gamma \mid Y}(\gamma \mid y) \cdot f_{Y}(y) d y .
$$

But with lognormal distribution there is no closed form solution for $f_{\gamma}(\gamma)$ for the envelope of the received signal power. As shown in [1], the two parameter Gamma pdf can be used as a suitable substitute for lognormal pdf. From [20] the Gamma pdf can be given in terms of average SNR $\gamma_{0}$ as,

$$
f_{Y}(y)=\frac{1}{\Gamma(c)} \gamma_{0}^{-c} y^{c-1} \exp \left(-\frac{y}{\gamma_{0}}\right) ; y \geq 0,
$$

where $c$ is the shape parameter of the Gamma distribution and $\Gamma($. is the gamma function [21].

Considering the envelope of the signal to follow the Rayleigh distribution and the power of the envelope to follow the Gamma distribution given by eqn. (3), the channel as given in [20] can be modelled as the Rayleigh-Gamma or $K$ distributed channel given as a function of $\operatorname{SNR}(\gamma)$ as,

$$
f_{X}(\gamma)=\frac{2}{\gamma_{0} \Gamma(c)}\left(\frac{\gamma}{\gamma_{0}}\right)^{\frac{c-1}{2}} K_{c-1}\left(2 \sqrt{\frac{\gamma}{\gamma_{0}}}\right) ; \gamma \geq 0,
$$

where $K_{a}($.$) is the the modified Bessel function of the second$ kind. The $K$ fading channel has been generally used for RADAR and satellite applications [22] 23]. As is evident from eqn.(4), for the $K$ distribution; the severity of the short term fading cannot be changed. Hence the $K$ fading channel is not suitable for fading analysis where the fading characteristics vary. Thus for terrestrial applications a more general channel model is required.

The Nakagami-Gamma or $K_{G}$ channel model can be used for more generalized estimation of the combined effects of short-term and long-term fading. The envelope of the received signal follows the Nakagami- $m$ distribution and the power of the envelope follows the Gamma distribution. The $K_{G}$ fading distribution function, as shown in [1, 20], can be given as a function of SNR $(\gamma)$ as,

$f_{X}(\gamma)=\frac{2 \gamma_{0}}{\Gamma(c) \Gamma(m)} m^{m} \gamma^{m-1}\left(m \gamma_{0} \gamma\right)^{\frac{c-m}{2}} K_{c-m}\left(2 \sqrt{\frac{m \gamma}{\gamma_{0}}}\right) ; \gamma \geq 0$,

where $m$ is the Nakagami shape parameter and $m \geq 0.5$. From [24] $K_{a}($.$) is related to the Meijer-G function G_{p q}^{m n}($.$) by,$

$$
x^{\mu} K_{\nu}(x)=G_{02}^{20}\left(\begin{array}{l|c}
\frac{1}{4} x^{2} & - \\
\frac{1}{2} \mu+\frac{1}{2} \nu, \frac{1}{2} \mu-\frac{1}{2} \nu
\end{array}\right) .
$$

To facilitate calculations we use eqn.(6) in eqn.(5) and rewrite the $K_{G}$ distribution function as

$$
f_{X}(\gamma)=\frac{1}{\Gamma(c) \Gamma(m)} \frac{m}{\gamma_{0}} G_{02}^{20}\left(\frac{m \gamma}{\gamma_{0}} \mid \begin{array}{c}
- \\
c-1, m-1
\end{array}\right) ; \gamma \geq 0
$$

\section{SER CALCULATION OF STAR MQAM IN $K$ AND GENERALIZED- $K$ FADING CHANNELS}

Using the simple geometric method proposed by Craig in [25], closed form expressions for symbol error rate (SER) of dual ring 
$M$-ary Star QAM in AWGN channel is derived in [16] as,

$$
\left.P_{e}(\gamma)=\frac{1}{2 \pi} \sum_{k=1}^{4} \int_{0}^{\theta_{k}} \exp [-A(\theta, k) \cdot \gamma]\right] d \theta
$$

where,

$$
A(\theta, k)=\frac{\alpha_{k} M \sin ^{2} \phi_{k}}{2\left(1+L^{2}\right) \sin ^{2}\left(\phi_{k}+\theta\right)},
$$

and $\alpha_{k}, \phi_{k}$ and $\theta_{k}$ are geometry dependent parameters given in [16]. $M$ is the total number of signalling points on the constellation and $\mathrm{L}$ is the ring ratio [16, 17]. $\alpha_{k}, \phi_{k}$ and $\theta_{k}$ are either constants or functions of $M$ and $L$. The expression put forth in [16] can be easily computed by numerical method and gives an exact approximation of the SER for lower order $M$-ary Star QAM schemes.

The SER, $P_{e}(\gamma)$, in a fading channel characterized by the fading distribution $f_{X}(\gamma)$, is given by,

$$
P(\gamma)=\int_{0}^{\infty} P_{e}(\gamma) \cdot f_{X}(\gamma) \cdot d \gamma
$$

\subsection{SER in Rayleigh-Gamma $(K)$ Fading Channel}

The average SER in the Rayleigh-Gamma $(K)$ channel can be obtained by substituting eqn. (4) and eqn.(8) in eqn.(10). Thus the average SER in the $K$ channel is the solution of integral given by,

$$
\begin{aligned}
\left.P_{e}\left(\gamma_{0}\right)\right|_{K}= & \frac{1}{2 \pi} \sum_{k=1}^{k=4} \int_{0}^{\theta_{k}} \int_{0}^{\infty} \frac{2}{\gamma_{0} \Gamma(c)}\left(\frac{\gamma}{\gamma_{0}}\right)^{\frac{c-1}{2}} \\
& \times K_{c-1}\left(2 \sqrt{\frac{\gamma}{\gamma_{0}}}\right) \times \exp (A(\theta, k) \gamma) d \gamma d \theta
\end{aligned}
$$

Using the relation [24]

$$
\begin{aligned}
\int_{0}^{\infty} x^{\frac{m}{2}} e^{-\alpha x} & K_{m}(2 \sqrt{x})= \\
& \frac{\Gamma(m+1)}{2 \alpha}\left(\frac{1}{\alpha}\right)^{\frac{m-1}{2}} \cdot W_{-\frac{1}{2} c,-\frac{1}{2}(c-1)}\left(\frac{1}{\alpha}\right)
\end{aligned}
$$

to compute the inner most integral of eqn.11, the final closed form expression for SER in $K$ fading channel is given as,

$$
\begin{aligned}
\left.P_{e}\left(\gamma_{0}\right)\right|_{K}= & \frac{1}{2 \pi} \sum_{k=1}^{k=4} \int_{0}^{\theta_{k}}\left(\frac{1}{A(\theta, k) \gamma_{0}}\right) \\
& \times \exp \left(\frac{1}{2 A(\theta, k) \gamma_{0}}\right) \\
& \times W_{-\frac{1}{2} c,-\frac{1}{2}(c-1)}\left(\frac{1}{A(\theta, k) \gamma_{0}}\right) d \theta,
\end{aligned}
$$

where $W_{a, b}(z)$ is the Whittaker function.

\subsection{SER in Nakagami-Gamma $\left(K_{G}\right)$ Fading Channel}

The average SER in Nakagami-Gamma $\left(K_{G}\right)$ fading channel can be computed by substituting eqn.(7) and eqn. 8 in eqn.(10). The average SER is the solution of the integral given by,

$$
\begin{aligned}
\left.P_{e}\left(\gamma_{0}\right)\right|_{K_{G}}= & \frac{1}{2 \pi} \sum_{k=1}^{k=4} \int_{0}^{\theta_{k}} \int_{0}^{\infty} \frac{1}{\Gamma(c) \Gamma(m)} \\
& \times\left(\frac{m}{\gamma_{0}}\right) \exp (A(\theta, k) \gamma) \\
& \times G_{02}^{20}\left(\left.\frac{m \gamma}{\gamma_{0}}\right|_{c-1, m-1} ^{-}\right) d \gamma d \theta .
\end{aligned}
$$

Using the relation [24]

$$
\begin{aligned}
\int_{0}^{\infty} x^{-\rho} e^{-\beta x} G_{p q}^{m n}\left(\alpha x \mid \begin{array}{c}
a_{1}, \ldots, a_{p} \\
b_{1}, \ldots, b_{q}
\end{array}\right)= \\
\beta^{\rho-1} G_{p+1 q}^{m n+1}\left(\alpha x \mid \begin{array}{c}
\rho, a_{1}, \ldots, a_{p} \\
b_{1}, \ldots, b_{q}
\end{array}\right)
\end{aligned}
$$

for computing the inner most integral, the closed form expression for SER in $K_{G}$ fading channel is given as,

$$
\begin{aligned}
\left.P_{e}\left(\gamma_{0}\right)\right|_{K_{G}}= & \frac{1}{2 \pi} \sum_{k=1}^{k=4} \int_{0}^{\theta_{k}} \frac{1}{\Gamma(c) \Gamma(m)} \cdot\left(\frac{m}{\gamma_{0}}\right) \\
& \times \frac{1}{A(\theta, k)} G_{12}^{21}\left(\frac{m}{\gamma_{0} A(\theta, k)} \mid \begin{array}{c}
0 \\
c-1, m-1
\end{array}\right) d \theta .
\end{aligned}
$$

Computing the average value of SER using eqn. (16) becomes computationally expensive in MATLAB 2012 because MATLAB exports the Meijer-G function from the MuPad utility. From [21] we get the relation

$$
\begin{aligned}
G_{12}^{21}\left(x \mid \begin{array}{c}
a \\
b, c
\end{array}\right) & =\Gamma(b-a+1) \Gamma(c-a+1) x^{\frac{1}{2}(b+c-1)} \\
& \times e^{\frac{1}{2} x} W_{k, m}(x),
\end{aligned}
$$

where, $k=a-0.5(b+c+1)$ and $m=0.5(b-c)$. Using eqn.17) we can rewrite eqn. 16, as,

$$
\begin{aligned}
\left.P_{e}\left(\gamma_{0}\right)\right|_{K_{G}}= & \frac{1}{2 \pi} \sum_{k=1}^{k=4} \int_{0}^{\theta_{k}}\left(\frac{m}{\gamma_{0} A(\theta, k)}\right)^{\frac{1}{2}(c+m-1)} \\
& \times \exp \left(\frac{m}{2 \gamma_{0} A(\theta, k)}\right) \\
& \times W_{-\frac{1}{2}(c+m-1),-\frac{1}{2}(c-m)}\left(\frac{m}{A(\theta, k) \gamma_{0}}\right) d \theta .
\end{aligned}
$$

As expected, putting $m=1$ in eqn.(31) we get eqn. (13). This is in conformance to the fact that the $K$ distribution is a special case of the $K_{G}$ distribution.

\section{BIT ERROR RATE ANALYSIS}

In [26] it is pointed out that traditional Gray's approximation underestimates the average bit error rate (BER) for Star QAM modulation. In fact Gray's approximation provides a lower bound (LB) for the BER. In this section we will derive a relation between the upper bound (UB) of BER and the SER using maximum $a$ priori probability estimate. 
Table 1. Average Asymptotical Optimum Ring Ratio in $K_{G}$ Fading Channel.

\begin{tabular}{ccc}
\hline \hline $\mathrm{M}$ & $L_{\text {opt }}$ in AWGN & Average $L_{\text {opt }}$ in $K_{G}$ \\
\hline 8 & 2.414 & 2.390 \\
16 & 1.765 & 1.805 \\
32 & 1.390 & 1.445 \\
64 & 1.196 & 1.255 \\
\hline
\end{tabular}

By maximum a priori probability (MAP) estimate the upper bound of BER is given by,

$$
P_{b}(u) \leq \frac{1}{\log _{2}(M)} \sum_{j=1}^{M} d\left(c_{j}, c_{u}\right) \cdot A_{j \mid u}
$$

where, $c_{u}$ is the symbol received and $c_{j}$ is the symbol decoded and

$$
A_{j \mid u}=P\left(c_{j} \text { isdecoded } \mid c_{u} \text { isreceived }\right)
$$

and $\mathrm{d}\left(c_{j}, c_{u}\right)$ is the Hamming distance between symbol $c_{j}$ and $c_{u}$. Under conditions of severe and moderate channel degradation it can be assumed that in case of a symbol error all the other $M-$ 1 symbols are equi-probable receiver output. Thus the probability that symbol $\mathrm{u}$ is sent and $\mathrm{j}$ is decoded is given as,

$$
A_{j \mid u}=\frac{S E R\left(\gamma_{0}\right)}{M-1}
$$

where $\operatorname{SER}\left(\gamma_{0}\right)$ is the symbol error rate as a function of average input $\operatorname{SNR}\left(\gamma_{0}\right)$ as given in $\operatorname{Section} 3$ The average $\operatorname{SER}\left(\gamma_{0}\right)$ is independent of $\mathrm{j}$. Thus eqn. (19) can be simplified as,

$$
P_{b}(u) \leq \frac{S E R\left(\gamma_{0}\right)}{(M-1) \log _{2} M} \sum_{j=1}^{M} d\left(c_{j}, c_{u}\right)
$$

Eqn. 22 can be further simplified by substituting,

$$
\sum_{j=1}^{M} d\left(c_{j}, c_{u}\right)=m \cdot 2^{m-1}
$$

where, $m=\log _{2}(M)$, the number of bits per symbol.

Substituting equation 23 in equation $22, P_{b}$ is given as,

$$
P_{b} \leq \frac{M}{2(M-1)} \operatorname{SER}\left(\gamma_{0}\right)
$$

Thus the average BER under conditions of severe and moderate channel degradation is given by,

$$
\frac{S E R\left(\gamma_{0}\right)}{\log _{2}(M)} \leq B E R \leq \frac{M}{2(M-1)} S E R\left(\gamma_{0}\right)
$$

\section{ESTIMATION OF RING RATIO (L)}

The ring ratio (L) is a crucial parameter in the design of $M$-ary Star QAM systems. In AWGN channel the optimum value of ring ratio can be simply derived by equating the distance between a constellation point and its neighbours, i.e. from Figure 1 we equate the distance between $s_{0}$ and $s_{1}$ and $s_{0}$ and $s_{8}$. The relation can be easily computed using geometric methods as given in [27] for 16-ary Star QAM. Generalizing the expression the optimum ring ratio in AWGN is given by,

$$
L_{\text {opt }}=2 \cdot \sin \left(\frac{2 \pi}{M}\right)-1
$$

For fading channels there is no direct formula to compute the optimum ring ratio (L). We need to employ the expressions for SER to estimate the optimum value of L. For a given value of $\operatorname{SNR}(\gamma)$, the value of $L$ for which SER is minimum is computed programmatically. This is repeated for different values of SNR $(\gamma)$. As shown in [17], the value of the optimum ring ratio assumes an asymptotic value for higher SNR. This asymptotic value is considered as the optimum value of L. Following this approach the asymptotic values $L_{\text {opt }}$ in the $K_{G}$ fading channel is computed.

The function under the integral in eqn. 31] is a hypergeometric function and hence a direct numerical search becomes computationally expensive and impractical. To overcome this a combination of numerical and empirical method is utilized to compute the asymptotic values of $L_{\text {opt }}$. Initially a few values of SNR are chosen over a large range $(0$ to $50 \mathrm{~dB})$. As we get an estimation of the asymptotic value of $L_{o p t}$ from this set of data, we decrease our range and take equal number of data points over that range. For example if from the first test we see that the optimum value of $L$ starts assuming asymptotic values from $30 \mathrm{~dB}$, then in the next iteration we will decrease our range to $25 \mathrm{~dB}$ to $40 \mathrm{~dB}$ and find the variation of $L$ for minimum SER in this range. Repeating these steps we can calculate the accurate value of $L_{o p t}$ for the $K_{G}$ fading channel.

Obviously the value of $L_{o p t}$ varies with $c$ and $m$. In order to facilitate system design an average value of $L_{\text {opt }}$ has been proposed for each value of $M$. The values of $L_{\text {opt }}$ for different values of $c$ and $m(1 \leq m \leq 5,1 \leq c \leq 5)$ are within a range of $\pm 10 \%$ of the proposed mean values given in Table 5

\section{RESULTS AND DISCUSSIONS}

To establish the analytical results of Section 3 extensive Monte Carlo simulations are preformed using MATLAB. Independent and identically distributed generalized $\mathrm{K}$ variates can be easily generated by extending the method shown in [28] using Gamma variates. Let $G_{\theta}$ be a gamma distributed random variate with the probability density function,

$$
f_{X}(x ; \theta)=\Gamma^{-1}(\theta) x^{(\theta-1)} e^{x} .
$$

The generalized- $K$ random variates with parameters $m$ and $c$ can then be generated by,

$$
K_{G}=2 p \sqrt{\left(G_{m} \times G c\right)},
$$

where,

$$
p=\frac{1}{\sqrt{|c-m|+1}} .
$$

The $\operatorname{gamrnd}(\theta, 1, \mathrm{a}, \mathrm{b})$ function of the Statistical toolbox in MATLAB can be used to efficiently generate gamma variates.

The expressions derived in Section 3 are numerically evaluated by the adaptive Simpson quadrature formula using MATLAB 2012. From Fig 2 3, 4 and 5 it is evident that the expressions derived in Section 3 are in close agreement with the simulated results for a wide range of input SNR. From the plots it is evident that increasing $c$ and/or $m$ the SER decreases. This is at par with the general expectation as for higher values of $c$ and $m$, shadowing and fading respectively becomes less severe. 


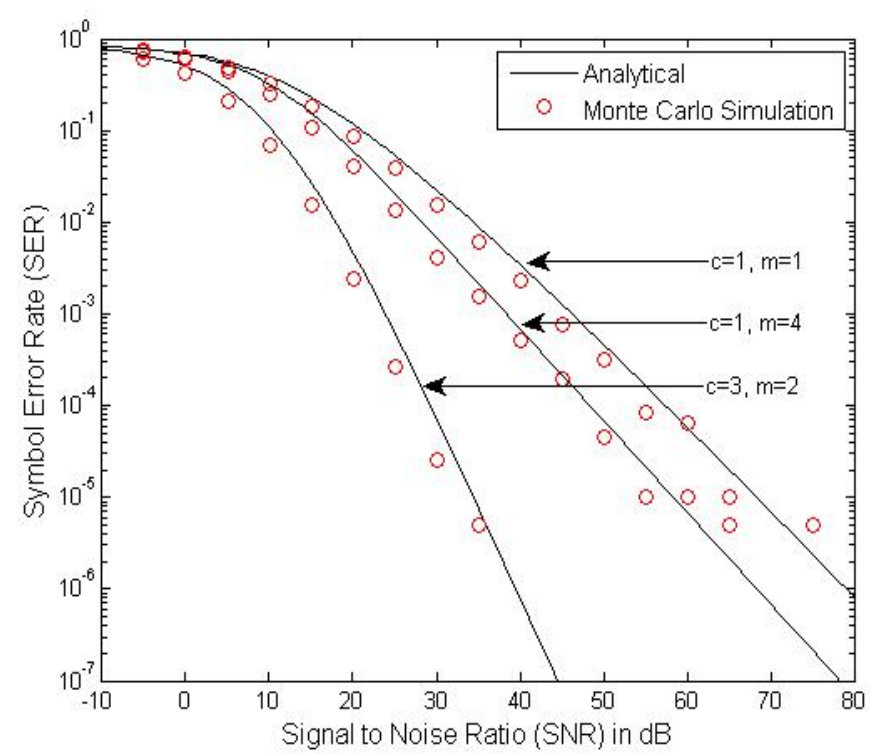

Fig. 2. Average SER vs. average SNR $\left(\gamma_{0}\right)$ of 8-ary Star QAM in Generalized- $K$ fading channel for several values of $m$ and $c(\mathrm{~L}=2.390)$.

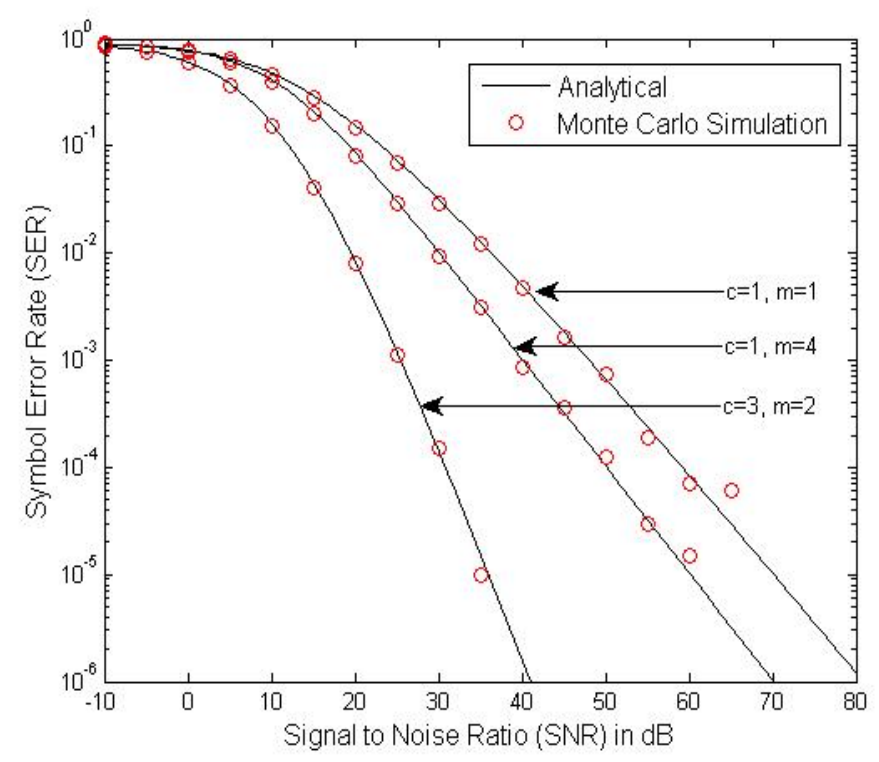

Fig. 3. Average SER vs. average SNR $\left(\gamma_{0}\right)$ of 16-ary Star QAM in Generalized- $K$ fading channel for several values of $m$ and $c(\mathrm{~L}=1.805)$.

From Figure 6 and Figure 7 it is seen that the estimation of BER using eqn. 25) is in close conformance to the Monte Carlo simulated results for high and moderate channel degradation. The importance of this result lies in the fact that BER is generally considered as the figure of merit of communication systems. To the best of the authors' knowledge, similar analysis for the circular QAM family is absent in current literature.

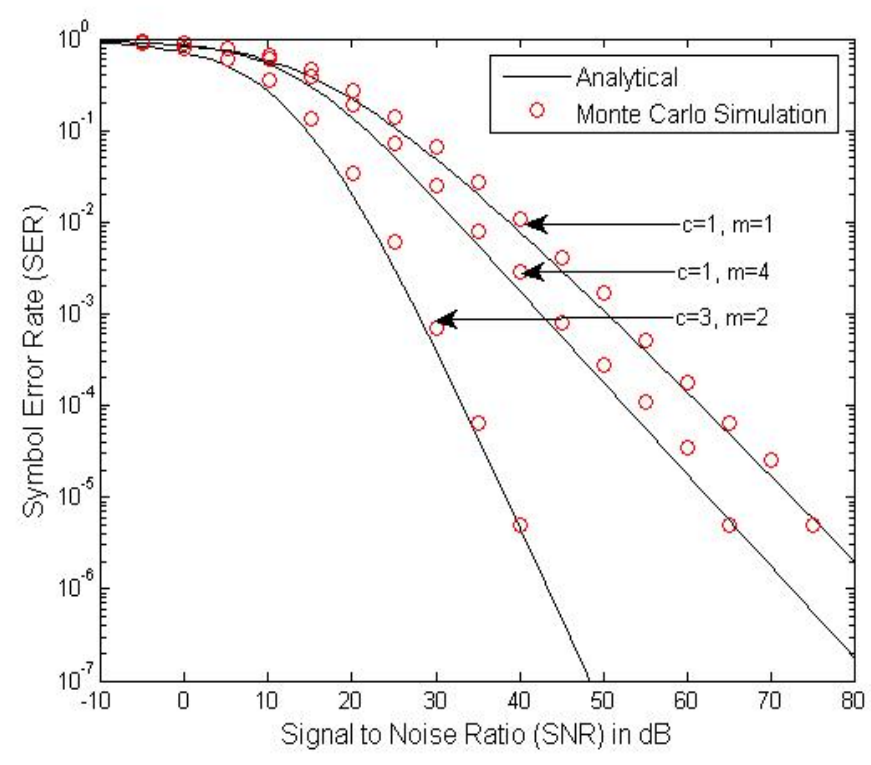

Fig. 4. Average SER vs. average SNR $\left(\gamma_{0}\right)$ of 32-ary Star QAM in Generalized- $K$ fading channel for several values of $m$ and $c(\mathrm{~L}=1.445)$.

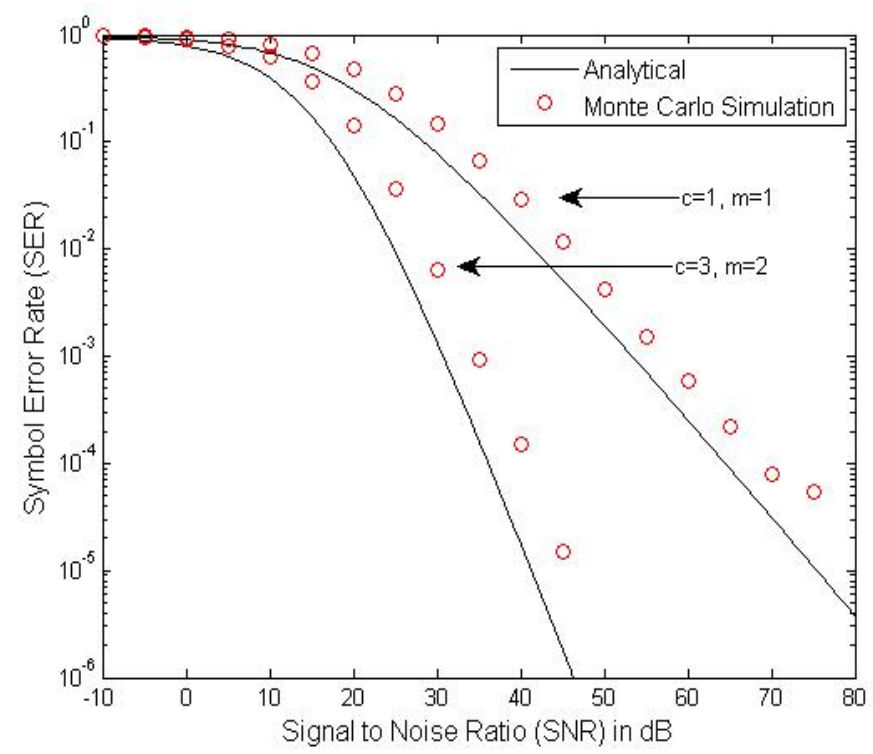

Fig. 5. Average SER vs. average SNR $\left(\gamma_{0}\right)$ of 64-ary Star QAM in Generalized- $K$ fading channel for several values of $m$ and $c(\mathrm{~L}=1.255)$.

\subsection{Comparison with Square $M Q A M$}

The SER of MQAM has been computed in [3] but the final expression in terms of the Meijer-G function is difficult to compute using MATLAB 2012. A close approximation of bit error rate (BER) for MQAM in AWGN channel has been given in [29] as,

$$
\operatorname{BER}_{M Q A M}(\gamma) \approx 0.2 \exp \left(-\frac{1.6 \gamma}{M-1}\right)
$$




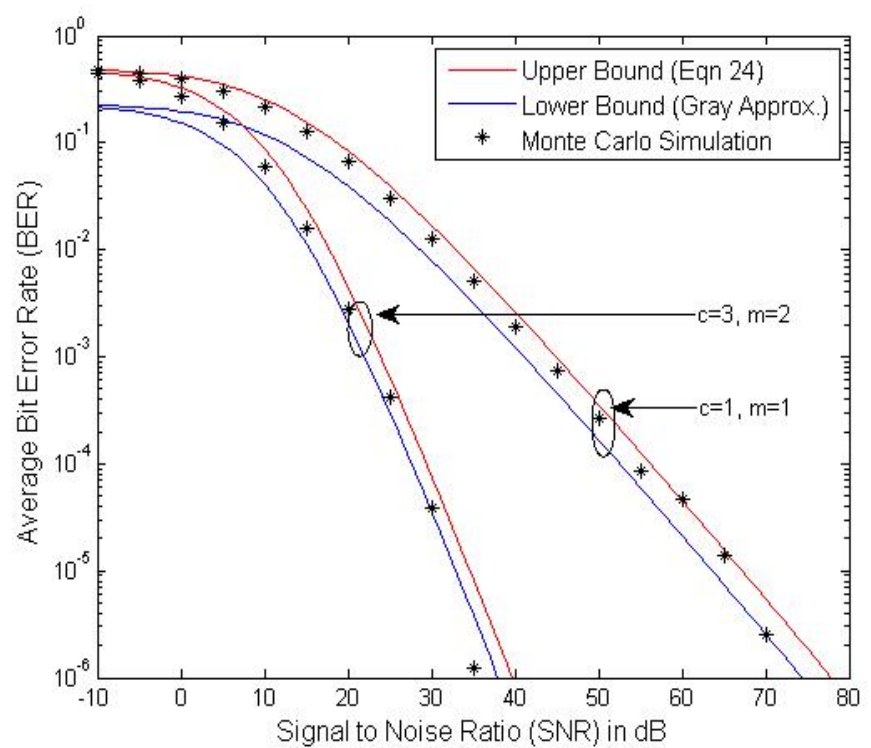

Fig. 6. UB and LB of average BER vs. average SNR $\left(\gamma_{0}\right)$ of 16-ary Star QAM in Generalized- $K$ fading channel for high and moderate channel degradation $(\mathrm{L}=1.805)$.

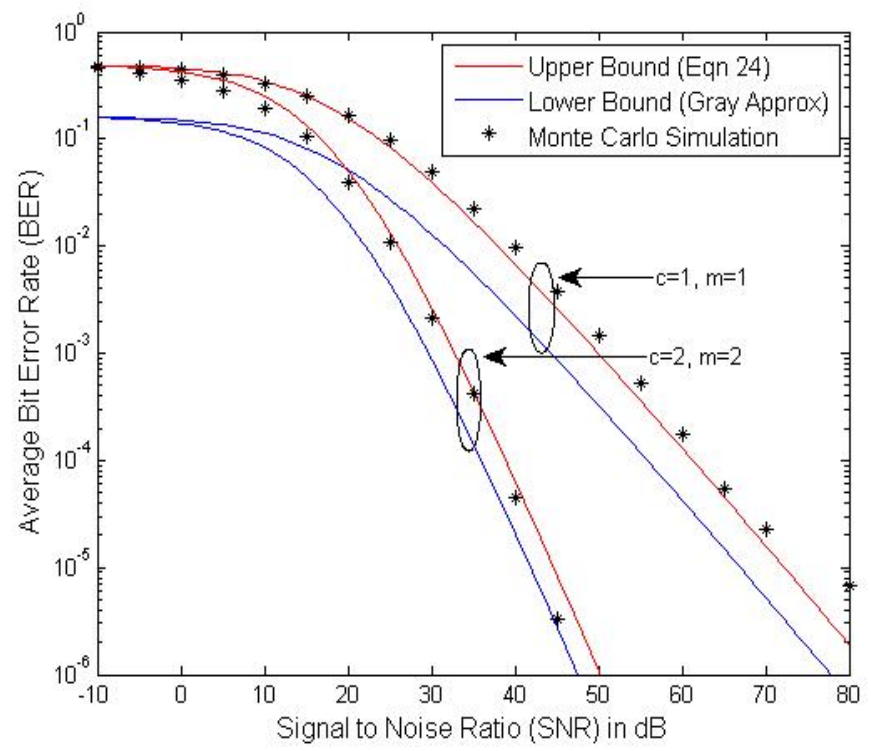

Fig. 7. UB and LB of average BER vs. average SNR $\left(\gamma_{0}\right)$ of 64-ary Star QAM in Generalized- $K$ fading channel for high and moderate channel degradation $(\mathrm{L}=1.255)$.

Following similar steps as in Section 3.2 we get the expression for BER of square $M \mathrm{QAM}$ in $K_{G}$ fading channel as,

$$
\begin{aligned}
\left.\operatorname{BER} R_{M Q A M}\left(\gamma_{0}\right)\right|_{K_{G}}= & .4\left(m \frac{M-1}{1.6 \gamma_{0}}\right)^{\frac{1}{2}(c+m-1)} \\
& \times \exp \left(\frac{1}{2} \frac{m(M-1)}{1.6 \gamma_{0}}\right) \\
& \times W_{-\frac{1}{2}(c+m-1),-\frac{1}{2}(c-m)}\left(m \frac{M-1}{1.6 \gamma_{0}}\right) d \theta .
\end{aligned}
$$

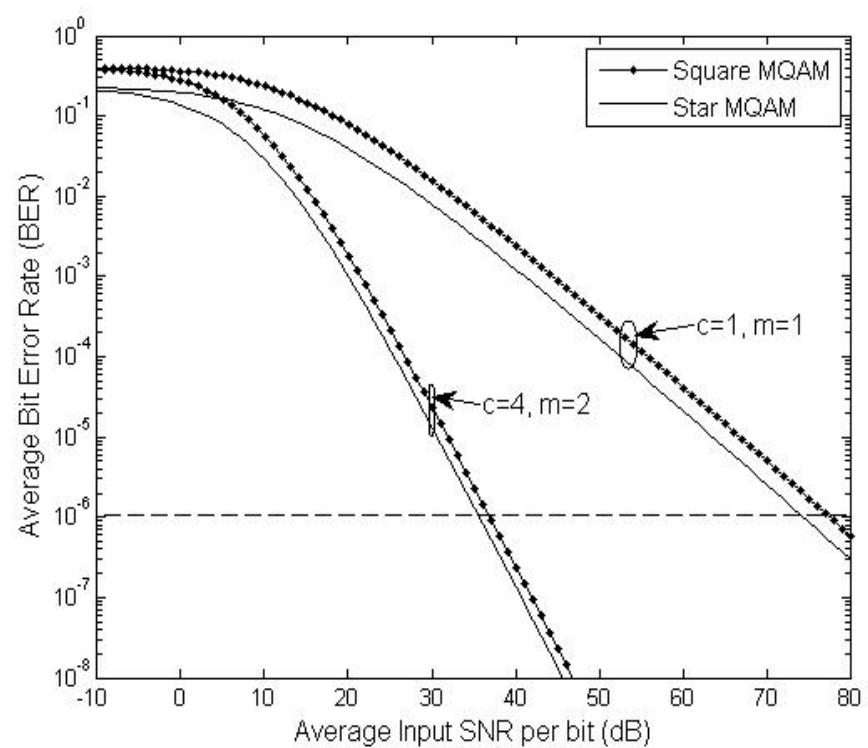

Fig. 8. Comparison of Error performance of 16-ary Star and Square QAM in $K_{G}$ Fading Channel for varying channel conditions ( $\mathrm{L}=1.805$ for Star QAM ).

The BER of Star MQAM can be estimated by the analytical expressions presented in Section 4

In our comparitive analysis we have considered the target BER to be $10^{-6}$. From Figure 8 it is seen that for $m=1$ and $c=1$ an advantage of $2.10 \mathrm{~dB}$ is achieved by the use of Star MQAM. Whereas for $m=2$ and $c=4$ the SNR advantage over Square $M$ QAM is $0.95 \mathrm{~dB}$. From Figure 9 we find that for 64 -ary schemes the advantage provided by Star $M$ QAM over Square MQAM is $6 \mathrm{~dB}$ for $m=1$ and $c=1$ and $3 \mathrm{~dB}$ for $m=2$ and $c=4$.

In light of the results it is contend that the Star MQAM scheme gives a considerable advantage over the popularly used Square $M$ QAM scheme in conditions of high and moderate fading. The advantage decrease with the decrease in the amount of fading (increase in $m$ or $c$ ). For very high values of $m$ and $c$ (i.e. for light fading) the Square MQAM has a better error performance. It can be noted that the results discussed here are similar in trend as the results discussed in [9, 17] for short-term fading channels.

\section{CONCLUSION}

The error rate performance of coherent $M$-ary 2-tier Star QAM signalling under long-term fading conditions is presented in this paper. The expressions are in terms of finite summation of single definite integrals of hypergeometric functions which can be computed using numerical techniques. The paper presents a novel approach to calculating the bit error rate of the Star QAM scheme from the expressions of SER. The paper also establishes the fact that Star MQAM signalling has better error performance than Square $M$ QAM in the $K$ and $K_{G}$ fading channels. The Star $M$ QAM is considerably better than the Square constellation for high and moderate fading. It can also be noted that higher order Star $M$ QAM gives greater advantage than its Square counterpart. Thus it can be inferred from our analysis that Star MQAM modulation is the better option than $M$-ary Square QAM for high rate data transmission over shadowed fading channels. 


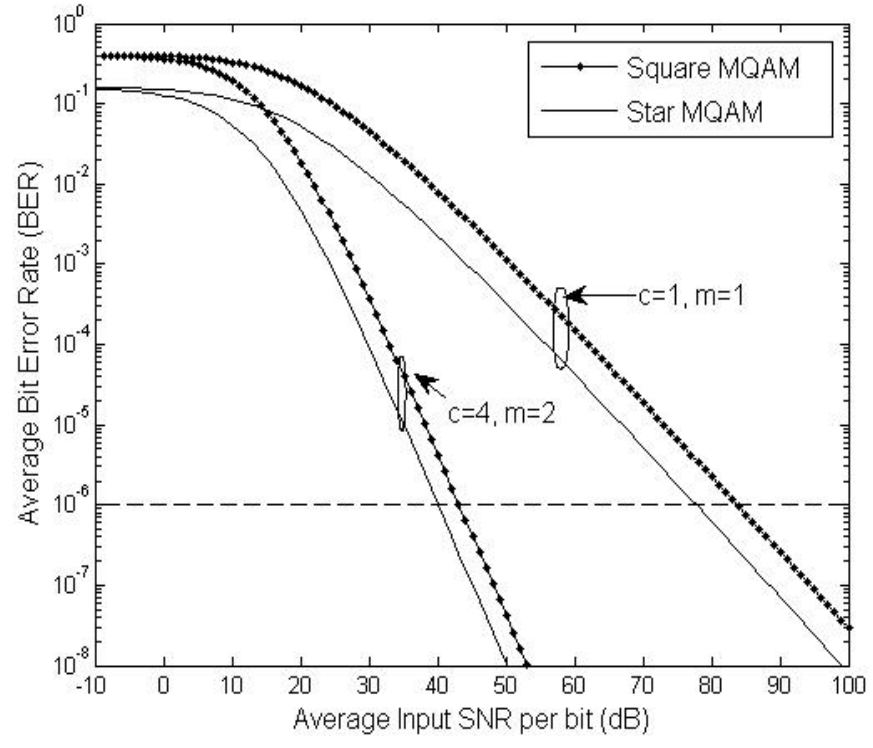

Fig. 9. Comparison of Error performance of 64-ary Star and Square QAM in $K_{G}$ Fading Channel for varying channel conditions ( $\mathrm{L}=1.255$ for Star QAM ).

\section{REFERENCES}

[1] P Mohana Shankar. Error rates in generalized shadowed fading channels. Wirel. Personal Commun., 28(3):233-238, 2004.

[2] A Abdi, H Allen Barger, and M Kaveh. A simple alternative to the lognormal model of shadow fading in terrestrial and satellite channels. In Proceedings of IEEE VTS 53rd Vehicular Technology Conference (VTC 2001), pages 2058-2062, Rhodes, Greece, 2001. IEEE.

[3] Petros S Bithas, Nikos C Sagias, P Takis Mathiopoulos, George K Karagiannidis, and Athanasios A Rontogiannis. On the performance analysis of digital communications over generalized-K fading channels. IEEE Commun. Lett., 10(5):353-355, 2006.

[4] TA Tsiftsis. Performance of heterodyne wireless optical communication systems over gamma-gamma atmospheric turbulence channels. Electron. Lett., 44(5):373-375, 2008.

[5] HE Nistazakis, TA Tsiftsis, and GS Tombras. Performance analysis of free-space optical communication systems over atmospheric turbulence channels. IET Commun., 3(8):1402-1409, 2009.

[6] Ali Abdi and Mostafa Kaveh. Comparison of DPSK and MSK bit error rates for $\mathrm{K}$ and Rayleigh-lognormal fading distributions. IEEE Commun. Lett., 4(4):122-124, 2000.

[7] George P Efthymoglou, Nikolaos Bissias, and Valentine A Aalo. On the error rate analysis of dual-hop amplify-and-forward relaying in generalized-K fading channels. J. Electr. Comput. Eng., 2010:31, 2010.

[8] PR Gould, WT Webb, and R Steele. Communications to the home via Star QAM. In Proceedings of IEE Colloquium on Customer Access-the Last 1.6 km, pages 7-1, London, 1993. IET.
[9] W.T.Webb and R. Steele. Variable rate QAM for mobile radio. IEEE Trans. Commun., 43(7):2223-2230, 1995.

[10] N. Wu, H. Wang, J. M. Kuang, and C. X. Yan. Performance analysis and design optimization of non-data-aided carrier frequency estimator for APSK signals. IEICE Trans. Commun., E95-B(6):2080-2086, June 2012.

[11] Mariia Sorokina, Stylianos Sygletos, Andrew D Ellis, and Sergei Turitsyn. Optimal packing for cascaded regenerative transmission based on phase sensitive amplifiers. Optics Express, 21(25):31201-31211, 2013.

[12] Noriko Iiyama, Jun-ichi Kani, Jun Terada, and Naoto Yoshimoto. Feasibility study on a scheme for coexistence of dsp-based PON and 10-gbps/ $\lambda$ PON using hierarchical star QAM format. J. Lightwave Technology, 31(18):3085-3092, 2013.

[13] Hongyu Zhang, Pooi-Yuen Kam, and Changyuan Yu. Optimal ring ratio of 16-star quadrature amplitude modulation in coherent optical communication systems. In Proceedings of 16th OptoeElectronics and Communications Conference (OECC), 2011, pages 577-578, Kaohsiung, 2011. IEEE.

[14] Hongyu Zhang, Pooi-Yuen Kam, and Changyuan Yu. Performance analysis of coherent optical 8-star QAM systems using decision-aided maximum likelihood phase estimation. Optics Express, 20(8):9302-9311, 2012.

[15] Youngjun Kim and Young-Chai Ko. Detection of quantum circular QAM signals. In Proceedings of the International Conference on ICT Convergence (ICTC), 2013, pages 1078-1082, Jeju Island, Korea (South), October 2013. IEEE.

[16] Sourjya Dutta and Aniruddha Chandra. Accurate SER expressions for $M$-ary dual ring star QAM in fading channels. In Proceedings of International Conference on Communications, Devices and Intelligent Systems (CODIS), 2012, pages 1-4, Kolkata, India, 2012. IEEE.

[17] Xiaodai Dong, Norman C Beaulieu, and Paul H Wittke. Error probabilities of two-dimensional $\mathrm{M}$-ary signaling in fading. IEEE Trans. Commun., 47(3):352-355, 1999.

[18] I. S. Barbounakis and A. M. Papadakis. Closed-form SER expressions for star MQAM in frequency non-selective Rician and Nakagami- $m$ channels. Int. J. Electron. Commun., 59(7):417-420, November 2005.

[19] Marvin K Simon and Mohamed Slim Alouini. A unified approach to the performance analysis of digital communication over generalized fading channels. Proc. IEEE, 86(9):1860-1877, 1998.

[20] Danijela Aleksić, Mihajlo Stefanović, Zoran Popović, Dragan Radenković, and Jovan D Ristić. On the $\mathrm{K}$ and $K_{G}$ fading channels. Serbian J. of Electr. Eng., 6(1):187-201, 2009.

[21] Harry Bateman and Arthur Erdélyi. Higher Transcendental Functions. Krieger, Melbourne, 1981.

[22] GA Lampropoulos, A Drosopoulos, N Rey, et al. High resolution radar clutter statistics. IEEE Trans. Aerosp. Electron. Syst., 35(1):43-60, 1999.

[23] D Lewinski. Nonstationary probabilistic target and clutter scattering models. IEEE Trans. Antennas Propag., 31(3):490-498, 1983

[24] Izrail S Gradshteyn and IM Ryzhik. Table of Integrals, Series and Products. Academic, New York, (1.421), 1980.

[25] J. W. Craig. A new, simple, and exact result for calculating the probability of error for two-dimensional signal constellations. In Proceedings of IEEE Military 
Communications Conference (MILCOM91), pages 571-575, McLean, VA, USA, October 1991.

[26] Lei Xiao and Xiaodai Dong. The exact transition probability and bit error probability of two-dimensional signaling. IEEE Trans. Wireless Commun., 4(5):2600-2609, 2005.

[27] L. N. Binh. Dual-ring 16-star QAM direct and coherent detection in $100 \mathrm{~Gb} / \mathrm{s}$ optically amplified fiber transmission: simulation. Opt. Quantum Electron., 40(10):707-732, August
2008.

[28] D Robert Iskander and Abdelhak M Zoubir. Estimation of the parameters of the k-distribution using higher order and fractional moments [radar clutter]. , IEEE Trans. Aerosp. Electron. Syst., 35(4):1453-1457, 1999.

[29] Seong Taek Chung and Andrea J Goldsmith. Degrees of freedom in adaptive modulation: a unified view. IEEE Trans. Commun., 49(9):1561-1571, 2001. 